\title{
Anosov Flows and Invariant Measures in Constrained Mechanical Systems
}

\author{
Waldyr M. Oliva ${ }^{1}$ \\ Instituto Superior Técnico, ISR and Departamento de Matemática, \\ Centro de Análise Matemática, Geometria e Sistemas Dinâmicos, \\ Av. Rovisco Pais, 1049-001 Lisboa, Portugal \\ E-mail address: wamoliva@math.ist.utl.pt
}

\section{Marcelo H. Kobayashi}

University of Hawai'i at Manoa, Department of Mechanical Engineering, 2540 Dole Street - Holmes Hall 302, Honolulu, HI 96822, USA

E-mail address: marcelok@hawaii.edu

\section{Gláucio Terra}

Universidade de São Paulo, Instituto de Matemática e Estatística, Departamento de Matemática,

Rua do Matão, 1010 05508-090 São Paulo, Brazil

E-mail address: glaucio@ime.usp.br

\begin{abstract}
We present conditions for hyperbolicity and existence of an invariant measure for the GMA flow of a non-linearly constrained mechanical system. The conservation of volume in the linear constrained problem corresponding to the rolling of a ball on a surface parallel to Delaunay is also considered.
\end{abstract}

\section{Introduction}

This paper concerns the flow of the GMA vector field of a non-linearly constrained mechanical system. Our main results are: (1) a condition for hyperbolicity, based on a Wojtkowski's criterion for hyperbolicity (see $[42]),(2)$ a condition for the existence of an invariant measure for such

\footnotetext{
${ }^{1}$ The first author wishes to thank Ana Rita Pires for all the fruitful discussions on Delaunay Sufaces and also to FCT (Portugal) for the support through the Program POCI 2010 and by the Project PDCT/MAT/56476/2004.
} 
a flow, which generalizes a theorem of Blackall ([5]) on the existence of invariant measures for linearly constrained systems and (3) the problem corresponding to the rolling of a ball on a parallel to a Delaunay surface. The constrained mechanical system is modelled by the following setup. We consider a smooth finite dimensional manifold $\mathrm{M}$, called the configuration space of the mechanical system, and a smooth function $\mathrm{K}: \mathrm{TM} \rightarrow \mathbb{R}$, called the kinetic energy, which we assume to be a positive definite quadratic form on each fiber of the velocity phase space TM. By polarization of this quadratic form on each fiber of TM, we obtain a smooth metric tensor $g$ on $M$, endowed of which it becomes a Riemannian manifold. The constraint is given by a smooth embedded submanifold $\mathscr{C}$ of the tangent bundle $\tau_{\mathrm{M}}$ : $\mathrm{TM} \rightarrow \mathrm{M}$, such that the restriction $\left.\tau_{\mathrm{M}}\right|_{\mathscr{C}}: \mathscr{C} \rightarrow \mathrm{M}$ is a submersion ${ }^{1}$. $\mathscr{C}$ is called the constraint manifold (or simply constraint). We say that the constraint is linear if $\mathscr{C}$ is a vector sub-bundle of $\mathrm{TM}^{2}$. A curve $\gamma$ on $\mathrm{M}$ is a motion or trajectory compatible with the constraint, or horizontal with respect to the constraint, if it is differentiable and its velocity lies in $\mathscr{C}$ almost everywhere on its domain. The dynamics of the mechanical system is given by a smooth fiber bundle morphism (i.e. it is a smooth map and preserves fibers) $\mathcal{F}: \mathrm{TM} \rightarrow \mathrm{T}^{*} \mathrm{M}$, called the external force. We say that the external force $\mathcal{F}$ derives from a potential $\mathrm{V} \in \mathfrak{F}(\mathrm{M})$ if it is of the form $v_{q} \in \mathrm{TM} \mapsto-d \mathrm{~V}(q) \in \mathrm{T}^{*} \mathrm{M}$.

In the unconstrained case, i.e. if $\mathscr{C}=\mathrm{TM}$, we say that a curve $\gamma$ on $\mathrm{M}$ is a motion or trajectory of the mechanical system $(\mathrm{M}, \mathrm{K}, \mathcal{F})$ if it is a solution of Newton's equation [33]:

$$
\mathcal{F}(\dot{\gamma})=\mu\left(\nabla_{t} \dot{\gamma}\right)
$$

where $\nabla$ is the Levi-Civita connection of the Riemannian manifold (M,g), $\nabla_{t}$ is the induced covariant derivative on fields along the curve $\gamma$ and $\mu=$ $\mathrm{g}^{b}: \mathrm{TM} \rightarrow \mathrm{T}^{*} \mathrm{M}$ is the Legendre transformation induced by the metric tensor. Using the notation $\mathrm{g}^{\sharp}:=\left(\mathrm{g}^{\mathrm{b}}\right)^{-1}: \mathrm{T}^{*} \mathrm{M} \rightarrow \mathrm{TM}$ and $\mathcal{F}^{\sharp}:=\mathrm{g}^{\sharp} \circ \mathcal{F}$ (which we also call external force), we obtain the following equivalent and more frequently used form of equation (1):

$$
\mathcal{F}^{\sharp}(\dot{\gamma})=\nabla_{t} \dot{\gamma}
$$

1. This is Marle's [28] definition of a "regular constraint". Other formulations of systems with non-linear constraints may be found in [38], [39], [37], [24], [25], [26], [3], [40], [31], [8], [28], [29], [30], [4], [9], [18], among others.

2. The linear constraint case is well known has an extensive literature ranging from classical texts such as [2], [41], [34] and [14] to a bibliography using modern differential geometry [7], [20], [12], [23], [6], [33] and [27], among others.

São Paulo J.Math.Sci. 2, 1 (2008), 55-76 
Taking vertical lifts on both members of the last equation, we obtain $\frac{T \dot{\gamma}}{d t}-\mathrm{H}_{\dot{\gamma}}(\dot{\gamma})=\lambda_{\dot{\gamma}}\left(\mathcal{F}^{\sharp}(\dot{\gamma})\right)$, showing that the solutions of (2) are the base integral curves (i.e. the projections on $\mathrm{M}$ of its integral curves) of the second order vector field $X_{\mathcal{F}} \in \mathfrak{X}(\mathrm{TM})$ defined by, for all $v_{q} \in \mathrm{TM}$, $X_{\mathcal{F}}\left(v_{q}\right)=\mathrm{S}\left(v_{q}\right)+\lambda_{v_{q}}\left(\mathcal{F}^{\sharp}\left(v_{q}\right)\right)$, where $\mathrm{S}$ is the geodesic spray of $(\mathrm{M}, \mathrm{g}) . X_{\mathcal{F}}$ is called the Gibbs-Maggi-Appell vector field (GMA) ${ }^{3}$ of $(\mathrm{M}, \mathrm{K}, \mathcal{F})$.

In the general case, we define a motion or trajectory of the constrained mechanical system $(\mathrm{M}, \mathrm{K}, \mathcal{F}, \mathscr{C})$ as a curve $\gamma$ on $\mathrm{M}$ which is compatible with the constraint $\mathscr{C}$ and which satisfies Newton's equation with reaction term $R$ :

$$
\nabla_{t} \dot{\gamma}=\mathcal{F}^{\sharp}(\dot{\gamma})+R(\dot{\gamma}),
$$

for some fiber preserving map $R: \mathscr{C} \rightarrow \mathrm{TM}$, called the reaction force field. We assume that $R$ is an admissible reaction in the sense of definition 5 , what ensures the existence of a second order vector field $X_{\mathscr{C}}^{R}$ on $\mathscr{C}$ (i.e. a vector field $X_{\mathscr{C}}^{R}: \mathscr{C} \rightarrow \mathrm{T} \mathscr{C}$ such that $\mathrm{T} \tau_{\mathrm{M}} \circ X_{\mathscr{C}}^{R}=\mathrm{id}_{\mathscr{C}}$ ) whose base integral curves are the solutions of (3). This vector field is obtained by taking vertical lifts on both members of equation (3).

As we can see in a previous paper (see [36]), a convenient choice $R=R^{A}$ of the admissible reaction force mentioned above, through a rule which generalizes d'Alembert's principle for linearly constrained systems, leads to the so called d'Alembert-Chetaev mechanics. The present paper focuses on the study of hyperbolicity and existence of invariant measures for the flow of the vector field $X_{\mathscr{C}}^{R^{A}}$ obtained by this choice of the reaction force, called the GMA vector field of the constrained mechanical system $(\mathrm{M}, \mathrm{K}, \mathcal{F}, \mathscr{C})$.

The organization of the paper is the following: in section 2 , we set up notation and recall some basic definitions from [36] concerning the geometry of the constraint manifold. In sections 3 and 4 (see theorems A and B) we prove, respectively, conditions for the GMA flow to be hyperbolic and to admit an invariant measure. Finally, in section 5 (see theorem $\mathrm{C}$ ) we study the conservation of a Riemannian volume under the GMA flow on a concrete example, given by the non-slipping rolling of a homogeneous ball over a parallel surface to a Delaunay surface in $\mathbb{R}^{3}$.

\section{Basic notations and definitions}

In this section we set up the notation and recall basic definitions concerning the geometry of the constraint manifold, parallel derivatives and d'Alembert-Chetaev trajectories. For more details, we refer the reader to

3. This nomenclature was suggested by Fusco and Oliva [12] in the context of linearly constrained mechanical systems. 
[36] (concerning the formulation of d'Alembert-Chetaev mechanics for nonlinearly constrained mechanical systems) and to [27] (for linearly constrained systems).

$M$ will denote a smooth connected finite dimensional manifold; TM (resp. $\mathbf{T}^{*} \mathrm{M}$ ) denotes the tangent (resp. cotangent) bundle of $\mathrm{M}$ and $\tau_{\mathrm{M}}: \mathrm{TM} \rightarrow \mathrm{M}$, $\tau_{\mathrm{M}}^{*}: \mathrm{T}^{*} \mathrm{M} \rightarrow \mathrm{M}$ the associated projections. We denote the trivial bundle over $\mathrm{M}$ with fiber $\mathrm{F}$ by $\mathrm{F}_{\mathrm{M}}$. In the present work, "smooth" means $C^{\infty}$. Following Helgason [15], the set of smooth functions on $M$, smooth vector fields on $M$ and Pfaffian forms on $M$ are denoted by $\mathfrak{F}(M), \mathfrak{X}(M)$ and $\mathfrak{U}_{1}(M)$, respectively. If $\pi_{E}: E \rightarrow \mathrm{M}$ is a smooth vector fiber bundle over $\mathrm{M}$ then $\mathbb{O}_{E}$ will denote the zero section of $E$, that is, $\mathbb{O}_{E}=\left\{\mathbb{O}_{p}: p \in \mathrm{M}\right\}$, with $\mathbb{O}_{p}$ the zero vector of $E_{p}=\pi_{E}^{-1}[p], p \in \mathrm{M}$. The set of smooth sections of $\pi_{E}: E \rightarrow \mathrm{M}$ is denoted by $\Gamma^{\infty}(E)$.

Let $E \oplus_{\mathrm{M}} E$ denote the Whitney sum of $\pi_{E}: E \rightarrow \mathrm{M}$ with itself. The vertical lift is the map $\lambda^{E}: E \oplus_{\mathrm{M}} E \rightarrow \mathrm{T} E$ such that, for any $q \in \mathrm{M}$, $v_{q} \in E_{q}, \lambda_{v_{q}}^{E}=\lambda^{E}\left(v_{q}, \cdot\right): E_{q} \rightarrow \mathrm{T}_{v_{q}} E$ is the tangent map at $v_{q}$ of the inclusion $E_{q} \rightarrow E$, using the canonical identification $\mathrm{T}_{v_{q}}\left(E_{q}\right) \equiv E_{q}$. That is, for all $w_{q} \in E_{q}$, we have: $\lambda_{v_{q}}^{E}\left(w_{q}\right)=\left.\frac{T}{d t}\right|_{t=0}\left(v_{q}+t w_{q}\right)$.

The map $\lambda^{E}$ is a smooth VB-monomorphism defined on the smooth vector bundle $\operatorname{pr}_{1}: E \oplus_{M} E \rightarrow E$ whose image is the vertical sub-bundle $\operatorname{Ver}(E)=\operatorname{ker}\left(\mathrm{T} \pi_{E}\right)$.

Let $\nabla: \Gamma^{\infty}(E) \rightarrow \Gamma^{\infty}\left(\mathrm{T}^{*} \mathrm{M} \otimes E\right)$ (or $\nabla^{E}$, if there is a risk of confusion) denote a connection on $\pi_{E}: E \rightarrow \mathrm{M}$. That is, $\nabla$ is an $\mathbb{R}$-linear map which satisfies the condition that, for any $f \in \mathfrak{F}(M)$ and any $\sigma \in \Gamma^{\infty}(E)$ : $\nabla(f \sigma)=\mathrm{d} f \otimes \sigma+f \nabla \sigma$. The connection $\nabla$ gives rise to a smooth VBmorphism $\mathrm{H}^{E}: E \oplus_{\mathrm{M}} \mathrm{TM} \rightarrow \mathrm{T} E$ : for any $q \in \mathrm{M}, w_{q} \in E_{q}$ and $v_{q} \in$ $\mathrm{T}_{q} \mathrm{M}$, choose any smooth curve $\gamma:(-\epsilon, \epsilon) \rightarrow M, t \mapsto \gamma(t)$, such that $\frac{T \gamma}{d t}_{\left.\right|_{t=0}}=v_{q}$. Let $\tau_{\gamma}(t): E_{q} \rightarrow E_{\gamma(t)}$ be the parallel transport along $\gamma$ defined by the connection. Then the tangent vector at 0 of the smooth curve $t \in(-\epsilon, \epsilon) \rightarrow \tau_{\gamma}(t) w_{q}$ is independent of the choice of $\gamma-$ it depends only on the pair $\left(v_{q}, w_{q}\right)$. We denote it by $\mathrm{H}_{v_{q}}^{E}\left(w_{q}\right)=\mathrm{H}^{E}\left(v_{q}, w_{q}\right) . \mathrm{H}^{E}$ defines a VB-monomorphism of the smooth vector bundle $\mathrm{pr}_{1}: E \oplus_{\mathrm{M}} \mathrm{TM} \rightarrow E$ into $\tau_{E}: \mathrm{T} E \rightarrow E$. Its image $\operatorname{Hor}(E)$ is the horizontal sub-bundle induced by the connection. $\mathrm{H}^{E}\left(v_{q}, w_{q}\right)$ is called the horizontal lift of $w_{q}$ at $v_{q}$, and is the unique vector at $\operatorname{Hor}_{v_{q}}(E)$ which projects (through $\mathrm{T} \pi_{E}$ ) to the vector $w_{q} \in \mathrm{T}_{q} \mathrm{M}$.

The smooth vector bundle $\tau_{E}: \mathrm{T} E \rightarrow E$ is the Whitney $\operatorname{sum} \operatorname{Hor}(E) \oplus_{E}$ $\operatorname{Ver}(E)$ of its horizontal and vertical sub-bundles. 
With a connection we can define the connector $\kappa_{E}: \mathrm{T} E \rightarrow E$, which is a VB-epimorphism from $\tau_{E}: \mathrm{T} E \rightarrow E$ to $\pi_{E}: E \rightarrow M$ such that for each $X_{v_{q}} \in \mathrm{T} E, \kappa_{E}\left(X_{v_{q}}\right) \in E_{v_{q}}$ is the unique vector which satisfies:

$$
X_{v_{q}}=\mathrm{H}_{v_{q}}^{E}\left(\mathrm{~T} \pi_{E} \cdot X_{v_{q}}\right)+\lambda_{v_{q}}^{E}\left(\kappa_{E} \cdot X_{v_{q}}\right) .
$$

The main significance of the preceding operators is that they allow us to work with objects in $\mathrm{M}$ and $E$ instead of $\mathrm{T} E$. For example, let $u$ : $(-\epsilon, \epsilon) \rightarrow E$ be a differentiable curve and $\gamma:(-\epsilon, \epsilon) \rightarrow \mathrm{M}$ be its projection on $\mathrm{M}, \gamma=\pi_{E} \circ u$. Denoting by $\dot{u}:=\frac{T u}{d t}$ the tangent vector field along $u$, we have $\kappa_{E} \cdot \dot{u}=\nabla_{t} u$, where $\nabla_{t}$ is the covariant derivative along $\gamma$ associated to the connection $\nabla$. Therefore, we have the following modified version of equation (4):

$$
\dot{u}=\mathrm{H}_{u}(\dot{\gamma})+\lambda_{u}\left(\nabla_{t} u\right) .
$$

For the sake of simplicity, from now on we will omit the " $E$ " from the notation, using $\mathrm{H}, \lambda, \kappa$ instead of $\mathrm{H}^{E}, \lambda^{E}$ and $\kappa_{E}$, respectively, whenever there is no risk of confusion.

2.1. The fiber and parallel derivatives. Let $\pi_{E}: E \rightarrow \mathrm{M}$ and

$\pi_{F}: F \rightarrow \mathrm{N}$ be smooth vector bundles over $M$ and $\mathrm{N}$, respectively, and let $b: E \rightarrow F$ be a smooth fiber bundle morphism over $\tilde{b}: \mathrm{M} \rightarrow \mathrm{N}$. That is, $b$, $\tilde{b}$ are smooth maps such that $\pi_{F} \circ b=\tilde{b} \circ \pi_{E}$.

The concept of fiber derivative of $b$ is well known (see, for example, [1]); it is the fiber bundle morphism $\mathbb{F} b$ defined by:

$$
\mathbb{F} b: \begin{array}{ccc}
E & \longrightarrow \mathrm{L}\left(E, \tilde{b}^{*} F\right) \\
v_{q} & \longmapsto \mathbb{F} b\left(v_{q}\right)
\end{array},
$$

where $\tilde{b}^{*} F$ is the pull back vector bundle of $F$ by $\tilde{b}$ and, for all $w_{q} \in E_{q}$ :

$$
\mathbb{F} b\left(v_{q}\right) \cdot w_{q}:=\kappa_{F}^{V} \cdot \mathrm{T} b \cdot \lambda_{v_{q}}\left(w_{q}\right)=\left.\frac{d}{d t}\right|_{t=0} b\left(v_{q}+t w_{q}\right) \in F_{\tilde{b}(q)},
$$

where $\frac{d}{d t}$ denotes the derivative of the curve $t \mapsto b\left(v_{q}+t w q\right)$ on the linear space $F_{\tilde{b}(q)}$.

Given connections $\nabla^{E}$ and $\nabla^{F}$ on the vector bundles $\pi_{E}: E \rightarrow \mathrm{M}$ and $\pi_{F}: F \rightarrow \mathrm{N}$, respectively, we introduce in the following definition a dual concept to the fiber derivative of $b$ :

Definition 1. The smooth fiber bundle morphism $\mathbb{P} b: E \rightarrow \mathrm{L}\left(\mathrm{TM}, \tilde{b}^{*} F\right)$ given by, for all $v_{q} \in E$ and all $z_{q} \in \mathrm{T}_{q} \mathrm{M}$ :

$$
\mathbb{P} b\left(v_{q}\right) \cdot z_{q}:=\kappa_{F} \cdot \mathrm{T} b \cdot \mathrm{H}_{v_{q}}\left(z_{q}\right) \in F_{\tilde{b}(q)}
$$

is called the parallel derivative of $b$. 
The idea of introducing these objects is to use the globally defined "partial derivatives" $\mathbb{F} b$ and $\mathbb{P} b$ to compute the tangent map of $b$. The following formulae will be extensively used:

$$
\begin{aligned}
\mathrm{T} \pi_{F} \cdot \mathrm{T} b \cdot X_{v_{q}} & =\mathrm{T} \tilde{b} \cdot \mathrm{T} \pi_{E} \cdot X_{v_{q}}, \\
\kappa_{F} \cdot \mathrm{T} b \cdot X_{v_{q}} & =\mathbb{F} b\left(v_{q}\right) \cdot \kappa_{E} \cdot X_{v_{q}}+\mathbb{P} b\left(v_{q}\right) \cdot \mathrm{T} \pi_{F} \cdot X_{v_{q}} .
\end{aligned}
$$

so that, given a curve $\gamma$ in $\mathrm{M}$ and a differentiable section $X$ of $E$ along $\gamma$, we have:

$$
\nabla_{t}^{F}(b \circ X)=\mathbb{F} b(X) \cdot \nabla_{t}^{E} X+\mathbb{P} b(X) \cdot \dot{\gamma} .
$$

2.2. The geometry of the constraint manifold. In this subsection we describe some notation and facts concerning the geometry of the constraint manifold.

Definition 2 (Marle). A constraint on $\mathrm{M}$ is a smooth embedded submanifold $\mathscr{C}$ of TM such that the restriction to $\mathscr{C}$ of the projection of the tangent bundle $\tau_{\mathrm{M}}: \mathrm{TM} \rightarrow \mathrm{M}$, henceforth denoted by $\pi_{\mathscr{C}}$, is a submersion. The constraint is said to be linear if $\mathscr{C}$ is a smooth vector sub-bundle of TM; we use the symbol $\mathscr{D}$ to denote linear constraints.

Given $q \in \mathrm{M}$, we denote by $\mathscr{C}_{q}$ the embedded submanifold $\pi_{\mathscr{C}}^{-1}[q] \subset \mathrm{T}_{q} \mathrm{M}$. Since $\pi_{\mathscr{C}}: \mathscr{C} \rightarrow \mathrm{M}$ is a submersion, $\mathrm{T} \pi_{\mathscr{C}}: \mathrm{T} \mathscr{C} \rightarrow \mathrm{TM}$ is a smooth vector bundle epimorphism; then $\operatorname{ker} \mathrm{T} \pi_{\mathscr{C}}$ is a smooth vector subbundle of $\mathrm{T} \mathscr{C}$, denoted henceforth by $\operatorname{Ver}(\mathscr{C})$, and called the vertical subbundle of $\mathrm{T} \mathscr{C}$. This sub-bundle is integrable; indeed, for all $v_{q} \in \mathscr{C}$, we have $\mathrm{T}_{v_{q}}\left(\mathscr{C}_{q}\right)=\operatorname{Ver}_{v_{q}}(\mathscr{C})$. Given $v_{q} \in \mathscr{C}$, we call $C_{v_{q}}:=\kappa^{V} \cdot \operatorname{Ver}_{v_{q}}(\mathscr{C}) \subset \mathrm{T}_{q} \mathrm{M}$ the subspace of virtual velocities (following the nomenclature of [3]) at $v_{q}$; $C_{v_{q}}$ is the subspace of $\mathrm{T}_{q} \mathrm{M}$ which is the image of the tangent map at $v_{q}$ of the inclusion $\mathscr{C}_{q} \rightarrow \mathrm{T}_{q} \mathrm{M}$.

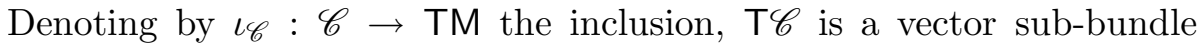
of the pull back vector bundle $\iota_{\mathscr{C}}^{*}$ TTM, also denoted by TTM $\left.\right|_{\mathscr{C}}$. Let us endow the vertical bundle $\operatorname{Ver}(\mathrm{TM})$ with the metric tensor induced by the metric $\mathrm{g}$ of $\mathrm{M}$ through the vertical lift, i.e. such that $\left(\forall v_{q} \in \mathrm{TM}\right) \lambda_{v_{q}}$ : $\mathrm{T}_{q} \mathrm{M} \rightarrow \operatorname{Ver}_{v_{q}}(\mathrm{TM})$ is a linear isometry. Since $\operatorname{Ver}(\mathscr{C})$ is a vector sub-bundle of the pull back $\iota_{\mathscr{C}}^{*} \operatorname{Ver}(\mathrm{TM})$, it makes sense to consider the orthogonal sub-bundle $W$ of $\operatorname{Ver}(\mathscr{C})$ in $i_{\mathscr{C}}^{*} \operatorname{Ver}(\mathrm{TM})$. That is to say, for all $v_{q} \in \mathscr{C}$, $W_{v_{q}}:=\operatorname{Ver}_{v_{q}}(\mathscr{C})^{\perp}$ is the orthogonal complement of $\operatorname{Ver}_{v_{q}}(\mathscr{C})$ in $\operatorname{Ver}_{v_{q}}(\mathrm{TM})$. The vector bundle $\pi_{W}: W \rightarrow \mathscr{C}$ is called the projection bundle ( $W$ is the pull back by the Legendre transformation $\mu$ of Marle's [28] projection bundle $W$ over $\left.D:=\mu(\mathscr{C}) \subset \mathrm{T}^{*} \mathrm{M}\right)$ on $\mathscr{C}$, induced by $\mathrm{g}$. For all $v_{q} \in \mathscr{C}$, the restrictions of the vertical lift at $v_{q}$ to $C_{v_{q}}$ and to its orthogonal complement 
are linear isometries: $\lambda_{v_{q}}: C_{v_{q}} \rightarrow \operatorname{Ver}_{v_{q}}(\mathscr{C})$ and $\lambda_{v_{q}}: C_{v_{q}}^{\perp} \rightarrow W_{v_{q}}$. We denote the orthogonal projections $\mathrm{T}_{q} \mathrm{M} \rightarrow C_{v_{q}}$ and $\mathrm{T}_{q} \mathrm{M} \rightarrow C_{v_{q}}^{\perp}$ by $\mathscr{P}\left(v_{q}\right)$ and $\mathscr{P}^{\perp}\left(v_{q}\right)$, respectively.

By the construction of $W$, we have the Whitney sum decomposition $i_{\mathscr{C}}^{*} \operatorname{Ver}(\mathrm{TM})=\operatorname{Ver}(\mathscr{C}) \oplus_{\mathscr{C}} W$. Besides, we also have the Whitney sum given by the following proposition $([28])$ :

Proposition 1. In the above situation, the following Whitney sum decomposition holds:

$$
i_{\mathscr{C}}^{*}(\mathrm{TTM})=\mathrm{T} \mathscr{C} \underset{\mathscr{C}}{\oplus} W
$$

We denote by $P_{\mathscr{C}}$ and $P_{W}$ the projections on the first and second factor of (5), respectively. Note that we have made use of the hypothesis of $\pi_{\mathscr{C}}$ being a submersion to construct the above splittings of TTM $\left.\right|_{\mathscr{C}}$ and $\left.\operatorname{Ver}(\mathrm{TM})\right|_{\mathscr{C}}$.

Let us now consider the Levi-Civita connection $\nabla$ of $(\mathrm{M}, \mathrm{g})$, and the corresponding horizontal sub-bundle $\operatorname{Hor}($ TM $) \subset$ TTM. We denote by $\operatorname{Hor}(\mathscr{C})$ the image by $P_{\mathscr{C}}$ of $\iota_{\mathscr{C}}^{*} \operatorname{Hor}(\mathrm{TM})$. We call Hor $(\mathscr{C})$ the horizontal sub-bundle of $\mathrm{T} \mathscr{C}$, induced by $\mathrm{g}$, and we have the following Whitney sum decomposition:

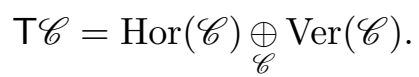

We denote by $P_{H}^{\mathscr{C}}: \mathrm{T} \mathscr{C} \rightarrow \operatorname{Hor}(\mathscr{C})$ and $P_{V}^{\mathscr{C}}: \mathrm{T} \mathscr{C} \rightarrow \operatorname{Ver}(\mathscr{C})$ the projections on the first and second factor of (6), respectively. Given $v_{q} \in \mathscr{C}$, we define the vertical and horizontal lifts in $\mathrm{T} \mathscr{C}, \lambda_{v_{q}}^{\mathscr{C}}:=\lambda_{v_{q}} \circ \mathscr{P}_{v_{q}}=P_{\mathscr{C}} \circ \lambda_{v_{q}}$ : $\mathrm{T}_{q} \mathrm{M} \rightarrow \operatorname{Ver}_{v_{q}}(\mathscr{C})$ and $\mathrm{H}_{v_{q}}^{\mathscr{C}}:=\left(\left.\mathrm{T} \tau_{\mathrm{M}}\right|_{\operatorname{Hor}_{v_{q}}(\mathscr{C})}\right)^{-1}=P_{\mathscr{C}} \circ \mathrm{H}_{v_{q}}: \mathrm{T}_{q} \mathrm{M} \rightarrow \operatorname{Hor}_{v_{q}}(\mathscr{C})$.

Note that, for all $v_{q} \in \mathscr{C}, \mathrm{H}_{v_{q}}^{\mathscr{C}}: \mathrm{T}_{q} \mathrm{M} \rightarrow \operatorname{Hor}_{v_{q}}(\mathscr{C})$ and $\left.\lambda_{v_{q}}^{\mathscr{C}}\right|_{C_{v_{q}}}: C_{v_{q}} \rightarrow \operatorname{Ver}_{v_{q}}(\mathscr{C})$ are linear isomorphisms. We can now use these linear isomorphisms to define a metric tensor on $\mathscr{C}$ through a construction which generalizes that of the definition of the Sasaki metric tensor on TM.

Definition 3 (The Sasaki metric tensor on $\mathscr{C}$ ). The Sasaki metric tensor or, simply, the Sasaki metric on $\mathscr{C}$ is the unique metric tensor $\mathrm{g}_{\mathscr{C}}$ on $\mathscr{C}$ such that, for all $v_{q} \in \mathscr{C},\left.\lambda_{v_{q}}^{\mathscr{C}}\right|_{C_{v_{q}}}: C_{v_{q}} \rightarrow \operatorname{Ver}_{v_{q}}(\mathscr{C})$ and $\mathrm{H}_{v_{q}}^{\mathscr{C}}: \mathrm{T}_{q} \mathrm{M} \rightarrow \operatorname{Hor}_{v_{q}}(\mathscr{C})$ are linear isometries.

In the case of a linear constraint $\mathscr{D}$, the Whitney sum decomposition (6) coincide with the one induced by the connection on $\mathscr{D}$ defined by $\nabla^{\mathscr{D}}: \mathfrak{X}(\mathrm{M}) \times \Gamma^{\infty}(\mathscr{D}) \rightarrow \Gamma^{\infty}(\mathscr{D}), \nabla_{X}^{\mathscr{D}} Y:=\mathscr{P}_{\mathscr{D}} \cdot \nabla_{X} Y$, where $\mathscr{P}_{\mathscr{D}}: \mathrm{TM} \rightarrow \mathscr{D}$ is the orthogonal projection. In that case, given $v_{q} \in \mathscr{D}, \lambda_{v_{q}}^{\mathscr{D}}$ and $\mathrm{H}_{v_{q}}^{\mathscr{D}}$ are the usual vertical and horizontal lifts at $v_{q}$, and we have $C_{v_{q}}=\mathscr{D}_{q}$, $W_{v_{q}}=\lambda_{v_{q}}\left(\mathscr{D}_{q}^{\perp}\right)$, so that $P_{\mathscr{D}}=\mathrm{T} \mathscr{P}_{\mathscr{D}}:\left.\mathrm{TTM}\right|_{\mathscr{C}} \rightarrow \mathscr{D}$. 
We define next the fiber and parallel derivatives for maps : $\mathscr{C} \rightarrow E$, where $\pi_{E}: E \rightarrow \mathrm{M}$ a smooth vector bundle, which preserve fibers. That is the case, for example, of the maps $\mathscr{P}, \mathscr{P}^{\perp}: \mathscr{C} \rightarrow \mathrm{L}(\mathrm{TM}, \mathrm{TM})$.

Definition 4. Let $\pi_{E}: E \rightarrow \mathrm{M}$ be a smooth vector bundle, endowed with a connection $\nabla^{E}$, and $f: \mathscr{C} \rightarrow E$ a smooth map such that, for all $q \in \mathrm{M}$, $f\left(\mathscr{C}_{q}\right) \subset E_{q}$. We define the fiber derivative $\mathbb{F} f: \mathscr{C} \rightarrow \mathrm{L}(\mathrm{TM}, E)$ and the parallel derivative $\mathbb{P} f: \mathscr{C} \rightarrow \mathrm{L}(\mathrm{TM}, E)$ by, for all $v_{q} \in \mathscr{C}:$

$$
\begin{aligned}
& \mathbb{F} f\left(v_{q}\right):=\kappa_{E} \circ \mathrm{T}_{v_{q}} f \circ \lambda_{v_{q}}^{\mathscr{C}} \in \mathrm{L}\left(\mathrm{T}_{q} \mathrm{M}, E_{q}\right), \\
& \mathbb{P} f\left(v_{q}\right):=\kappa_{E} \circ \mathrm{T}_{v_{q}} f \circ \mathrm{H}_{v_{q}}^{\mathscr{C}} \in \mathrm{L}\left(\mathrm{T}_{q} \mathrm{M}, E_{q}\right) .
\end{aligned}
$$

Therefore, given $v_{q} \in \mathscr{C}$ and $X_{v_{q}} \in \mathrm{T}_{v_{q}} \mathscr{C}$, we have:

$$
\kappa_{E} \cdot \mathrm{T}_{v_{q}} f \cdot X_{v_{q}}=\mathbb{F} f\left(v_{q}\right) \cdot \kappa \cdot X_{v_{q}}+\mathbb{P} f\left(v_{q}\right) \cdot \mathrm{T} \pi_{\mathscr{C}} \cdot X_{v_{q}},
$$

and $\mathbb{F} f\left(v_{q}\right) \cdot \kappa \cdot X_{v_{q}}=\mathbb{F} f\left(v_{q}\right) \cdot \mathscr{P}_{v_{q}} \cdot \kappa \cdot X_{v_{q}}$, i.e. $C_{v_{q}}^{\perp} \subset \operatorname{ker} \mathbb{F} f\left(v_{q}\right)$.

As a final remark, given $f \in \mathfrak{F}(T M)$, we use the notation $\mathbb{F}^{\sharp} f$ and $\mathbb{P}^{\sharp} f$ to denote, respectively, the maps $\mathrm{g}^{\sharp} \circ \mathbb{F} f: \mathrm{TM} \rightarrow \mathrm{TM}$ and $\mathrm{g}^{\sharp} \circ \mathbb{P} f: \mathrm{TM} \rightarrow \mathrm{TM}$, where $g^{\sharp}$ is the inverse of the Legendre transformation $g^{b}: T M \rightarrow T^{*} M$ induced by the metric tensor $\mathrm{g}$.

2.3. d'Alembert-Chetaev Trajectories. In this subsection we recall the definition of the d'Alembert-Chetaev trajectories of the constrained mechanical system $(\mathrm{M}, \mathrm{K}, \mathcal{F}, \mathscr{C})$. Firstly, we introduce the concept of admissible reaction.

DeFinition 5. We say that a continuous map $R: \mathscr{C} \rightarrow$ TM is an admissible reaction field for the constrained mechanical system $(\mathrm{M}, \mathrm{K}, \mathcal{F}, \mathscr{C})$ if it is fiber preserving and if there exists a second order vector field $X_{\mathscr{C}}^{R}$ on $\mathscr{C}$ whose maximal integral curves with fixed initial condition exist and are unique, and whose base integral curves are solutions of Newton's equation with reaction term (3).

We denote by $\mathfrak{R}$ the set of all admissible reaction fields for $(\mathrm{M}, \mathrm{K}, \mathcal{F}, \mathscr{C})$. If $R \in \mathfrak{R}$, we call the base integral curves of $X_{\mathscr{C}}^{R}$ the trajectories of the constrained mechanical system $(\mathrm{M}, \mathrm{K}, \mathcal{F}, \mathscr{C})$, induced by the admissible reaction $R$.

Remark 1. Note that, if $R$ is an admissible reaction field for $(\mathrm{M}, \mathrm{K}, \mathcal{F}, \mathscr{C})$, then $X_{\mathscr{C}}^{R}$ is univocally determined by $R$. In fact, taking vertical lifts in (3), it follows that $X_{\mathscr{C}}^{R}$ must be given by:

$$
\mathrm{S}\left(v_{q}\right)+\lambda_{v_{q}}\left\{\mathcal{F}^{\sharp}\left(v_{q}\right)+R\left(v_{q}\right)\right\},
$$

where $\mathrm{S}$ is the geodesic spray of $(\mathrm{M}, \mathrm{g})$ and $\mathcal{F}^{\sharp} \doteq \mathrm{g}^{\sharp} \circ \mathcal{F}: \mathrm{TM} \rightarrow \mathrm{TM}$. 
Let $X_{\mathcal{F}}: \mathrm{TM} \rightarrow \mathrm{T}(\mathrm{TM})$ be the GMA vector field of the unconstrained mechanical system $(M, K, \mathcal{F})$ (i.e. the second order vector field whose base integral curves are the solutions of Newton's equation (2)). That is to say, $X_{\mathcal{F}}: v_{q} \in \mathrm{TM} \mapsto \mathrm{S}\left(v_{q}\right)+\lambda_{v_{q}}\left\{\mathcal{F}^{\sharp}\left(v_{q}\right)\right\}$. Using the Whitney sum decomposition TTM $\left.\right|_{\mathscr{C}}=\mathrm{T} \mathscr{C} \oplus_{\mathscr{C}} W$, the restriction of $X_{\mathcal{F}}$ to the constraint manifold $\mathscr{C}$ splits into a sum $\left.X_{\mathcal{F}}\right|_{\mathscr{C}}=X_{\mathscr{C}}+X_{W}$, where $X_{\mathscr{C}}$ is a smooth second-order vector field on $\mathscr{C}$ and $X_{W}$ a smooth section of the projection bundle $W$.

DEFINITION 6. Using the notation above, we call the second order vector field $X_{\mathscr{C}} \in \mathfrak{X}(\mathscr{C})$ the Gibbs-Maggi-Appell (GMA) vector field of the constrained mechanical system $(\mathrm{M}, \mathrm{K}, \mathcal{F}, \mathscr{C})$. The base integral curves of the GMA vector field $X_{\mathscr{C}}$ are called the d'Alembert-Chetaev trajectories of $(\mathrm{M}, \mathrm{K}, \mathcal{F}, \mathscr{C})$.

It is shown in [36] that the GMA vector field of $(\mathrm{M}, \mathrm{K}, \mathcal{F}, \mathscr{C})$ is induced by an admissible reaction $R_{\mathcal{F}}^{A}$ that has the remarkable property of minimizing the intensity of the admissible reactions (i.e. it satisfies the Gauss principle of least constraint). This reaction is given by, for all $v_{q} \in \mathscr{C}$ :

$$
R_{\mathcal{F}}^{A}\left(v_{q}\right)=-\kappa \cdot P_{W} \cdot \mathrm{S}\left(v_{q}\right)-\mathscr{P}^{\perp}\left(v_{q}\right) \cdot\left(\mathcal{F}^{\sharp}\left(v_{q}\right)\right)
$$

so that $X_{\mathscr{C}}$ is given by (7) with $R$ replaced by $R_{\mathcal{F}}^{A}$ given by the formula above.

\section{A criterion for hyperbolicity}

In this section, we consider a constrained mechanical system $(\mathrm{M}, \mathrm{K}, \mathcal{F}, \mathscr{C})$ with $\mathrm{M}$ compact and establish sufficient conditions such that the flow associated with $X_{\mathscr{C}}\left(R_{\mathcal{F}}\right)$ be hyperbolic. Let us recall the definition of hyperbolic flow.

Definition 7. Let (M,g) be a Riemannian, compact manifold and $X \in$ $\mathfrak{X}(\mathrm{M})$ a vector field in $\mathrm{M}$ such that $X(q) \neq 0$, for all $q \in \mathrm{M}$. We denote by $\left(\phi^{t}\right)_{t \in \mathbb{R}}$ the flow of $X$. The flow $\phi^{t}$ is hyperbolic or Anosov if there exist $\mathrm{T} \phi^{t}$-invariant distributions on $\mathrm{M}: E^{0}, E^{+}$and $E^{-}$, such that:

(1) for all $q \in \mathrm{M}, \mathrm{T}_{q} \mathrm{M}=E_{q}^{0} \oplus E_{q}^{+} \oplus E_{q}^{-}$;

(2) for all $q \in \mathrm{M}, E_{q}^{0}=[X(q)]$, where $[X(q)]$ denotes the linear closure of $X(q)$;

(3) there exist constants $a, b>0$ such that, for all $q \in \mathrm{M}$ and all $t \geq 0$ we have:

$$
\begin{aligned}
\left\|\mathrm{T} \phi^{t} \cdot v\right\| & \leq b e^{-t a}\|v\|, \forall v \in E_{q}^{-} \\
\left\|\mathrm{T} \phi^{-t} \cdot v\right\| & \leq b e^{-t a}\|v\|, \forall v \in E_{q}^{+}
\end{aligned}
$$


The distributions $E^{0}, E^{+}$and $E^{-}$are called, respectively, neutral, unstable and stable.

It follows from the previous definition that $E^{+}$and $E^{-}$have constant rank in each connected component of $M$ and are continuous, that is, they are locally generated by local sections of $\tau_{\mathrm{M}}: \mathrm{TM} \rightarrow \mathrm{M}$.

DeFINITION 8. We denote by $\widehat{\mathrm{TM}}$ the quotient fiber bundle $\mathrm{TM} / E^{0}$, such that for all $q \in \mathrm{M}, \widehat{\mathrm{T}_{q} \mathrm{M}}=\mathrm{T}_{q} \mathrm{M} /[X(q)]$. Let $\pi: \mathrm{TM} \rightarrow \widehat{\mathrm{TM}}$ denote the canonical quotient projection. Assume there is a continuous, quadratic form $\mathrm{Q}: \mathrm{TM} \rightarrow \mathbb{R}$ (that is, a continuous function, which is a quadratic form in each fiber) satisfying the following conditions:

EM1 for all $q \in \mathrm{M}, v_{q} \in \mathrm{T}_{q} \mathrm{M}$ and $s \in \mathbb{R}$ we have $\mathrm{Q}\left(v_{q}+s X(q)\right)=\mathrm{Q}\left(v_{q}\right)$, so that there exists a continuous, quadratic form $\widehat{\mathrm{Q}}: \widehat{\mathrm{TM}} \rightarrow \mathrm{M}$ such that $\widehat{\mathrm{Q}} \circ \pi=\mathrm{Q}$;

EM2 the Lie derivative $\mathfrak{L}_{X} \mathrm{Q}: \mathrm{TM} \rightarrow \mathbb{R},\left(\forall v_{q} \in \mathrm{TM}\right) \mathfrak{L}_{X} \mathrm{Q}\left(v_{q}\right)=$ $\left.\frac{d}{d t}\right|_{t=0} \mathrm{Q}\left(\mathrm{T} \phi^{t} \cdot v_{q}\right)$, exists and is continuous. It follows from EM1 that there exits $\widehat{\mathfrak{L}_{X} \mathrm{Q}}: \widehat{\mathrm{TM}} \rightarrow \mathbb{R}$ such that $\widehat{\mathfrak{L}_{X} \mathrm{Q}} \circ \pi=\mathfrak{L}_{X} \mathrm{Q}$;

EM3 $\widehat{Q}$ in non-degenerate and $\widehat{\mathfrak{L}_{X} \mathrm{Q}}$ is positive definite.

If such a quadratic form $Q$ exits, we say that the flow $\left(\phi^{t}\right)_{t \in \mathbb{R}}$ is strictly monotone.

We will use the following theorem to establish the condition for hyperbolicity mentioned at the beginning of this section:

TheOREM (Wojtkowski [42]). If the flow $\left(\phi^{t}\right)_{t \in \mathbb{R}}$ is strictly monotone then it is hyperbolic.

The following result provides a criterion for the hyperbolicity of the GMA flow $\left(\phi^{t}\right)_{t \in \mathbb{R}}$ of $X_{\mathscr{C}}\left(R_{\mathcal{F}}\right)$ :

Theorem A. Let $\mathscr{M}$ be a compact, $\phi^{t}$-invariant submanifold of $\mathscr{C}$, with empty intersection with the the zero section of TM. Given $v_{q} \in \mathscr{C}$ and $v_{q} \neq 0$, consider the following quadratic forms defined on $\widehat{\mathrm{T}_{v_{q}} \mathscr{C}}$ : 


$$
\begin{aligned}
Q_{1}\left(v_{q}\right) & : X_{v_{q}} \mapsto\left\langle\kappa \cdot X_{v_{q}}, \mathrm{~T} \pi_{\mathscr{C}} \cdot X_{v_{q}}\right\rangle \\
Q_{2}\left(v_{q}\right) & : X_{v_{q}} \mapsto\left\langle\mathrm{T} \pi_{\mathscr{C}} \cdot X_{v_{q}}, \mathrm{R}\left(v_{q}, \mathrm{~T} \pi_{\mathscr{C}} \cdot X_{v_{q}}\right) \cdot v_{q}\right\rangle \\
& +\left\langle\mathrm{T} \pi_{\mathscr{C}} \cdot X_{v_{q}}, \mathbb{F} \mathcal{F}^{\sharp}\left(v_{q}\right) \cdot \kappa \cdot X_{v_{q}}+\mathbb{P} \mathcal{F}^{\sharp}\left(v_{q}\right) \cdot \mathrm{T} \pi_{\mathscr{C}} \cdot X_{v_{q}}\right\rangle \\
& +\left\langle\mathrm{T} \pi_{\mathscr{C}} \cdot X_{v_{q}}, \mathbb{F} R_{\mathcal{F}}\left(v_{q}\right) \cdot \kappa \cdot X_{v_{q}}+\mathbb{P} R_{\mathcal{F}}\left(v_{q}\right) \cdot \mathrm{T} \pi_{\mathscr{C}} \cdot X_{v_{q}}\right\rangle \\
& +\left\langle\kappa \cdot X_{v_{q}}, \kappa \cdot X_{v_{q}}\right\rangle \\
& +\left[2 \frac{\left\langle\mathcal{F}^{\sharp}\left(v_{q}\right)+R_{\mathcal{F}}\left(v_{q}\right), v_{q}\right\rangle}{\left\langle v_{q}, v_{q}\right\rangle^{2}}\right. \\
& \left.-\frac{\left\langle\kappa \cdot X_{v_{q}}, v_{q}\right\rangle+\left\langle\mathcal{F}^{\sharp}\left(v_{q}\right)+R_{\mathcal{F}}\left(v_{q}\right), \mathrm{T} \pi_{\mathscr{C}} \cdot X_{v_{q}}\right\rangle}{\left\langle v_{q}, v_{q}\right\rangle}\right]\left[\left\langle\cdot X_{v_{q}}, v_{q}\right\rangle\right. \\
& \left.+\left\langle\mathcal{F}^{\sharp}\left(v_{q}\right)+R_{\mathcal{F}}\left(v_{q}\right), \mathrm{T} \pi_{\mathscr{C}} \cdot X_{v_{q}}\right\rangle\right],
\end{aligned}
$$

where $\mathrm{R}$ is the curvature tensor associated with the Levi-Civita connection of $(\mathrm{M}, \mathrm{g})$.

If for all $v_{q} \in \mathscr{M}, Q_{1}\left(v_{q}\right)$ is non-degenerate and $Q_{2}\left(v_{q}\right)$ is positive definite, then the flow $\left(\phi^{t} \mid \mathscr{M}\right)_{t \in \mathbb{R}}$ of $\left.X_{\mathscr{C}}\left(R_{\mathcal{F}}\right)\right|_{\mathscr{M}}$ is hyperbolic.

In the proof of the previous theorem we will use the following lemma, which provides the equation of the Jacobi fields associated to the flow of $X_{\mathscr{C}}\left(R_{\mathcal{F}}\right)$ :

Lemma 1. Given $X_{v_{q}} \in \mathrm{T} \mathscr{C}$ we have, for all $t \in \mathbb{R}, \mathrm{T} \phi^{t} \cdot X_{v_{q}}=\mathrm{H}_{\dot{\gamma}(t)} J(t)+$ $\lambda_{\dot{\gamma}(t)} \nabla_{t} J(t)$, where $\dot{\gamma}=\phi^{t}\left(v_{q}\right)$ and $J \in \mathfrak{X}(\gamma)$ is a solution of

$$
\nabla_{t}^{2} J=\mathrm{R}(\dot{\gamma}, J) \cdot \dot{\gamma}+\mathbb{F} \mathcal{F}^{\sharp}(\dot{\gamma}) \cdot \nabla_{t} J+\mathbb{P F}^{\sharp}(\dot{\gamma}) \cdot J+\mathbb{F} R_{\mathcal{F}}(\dot{\gamma}) \cdot \nabla_{t} J+\mathbb{P} R_{\mathcal{F}}(\dot{\gamma}) \cdot J
$$

with initial condition $J(0)=\mathrm{T} \pi_{\mathscr{C}} \cdot X_{v_{q}}, \nabla_{t \mid t=0} J=\kappa \cdot X_{v_{q}}$.

Proof. Take $c:(-\epsilon, \epsilon) \rightarrow \mathscr{C}$ such that $c^{\prime}(0)=X_{v_{q}}$ and for all $t \in \mathbb{R}$, $s \in(-\epsilon, \epsilon)$ let $\gamma(t, s):=\pi_{\mathscr{C}} \circ \phi^{t} \circ c(s)$. Then, for each $s \in(-\epsilon, \epsilon), \gamma_{s}:=\gamma(\cdot, s)$ is a the integral base curve of the vector field $X_{\mathscr{C}}\left(R_{\mathcal{F}}\right)$, that is,

$$
\nabla_{t} \dot{\gamma}_{s}=\mathcal{F}^{\sharp}\left(\dot{\gamma}_{s}\right)+R_{\mathcal{F}}\left(\dot{\gamma}_{s}\right),
$$

and taking $\nabla_{s \mid s=0}$ to both sides of the previous equation, we obtain (9).

Proof of Theorem $A$. For $X_{\mathscr{C}}\left(R_{\mathcal{F}}\right)$ we can identify, for all $v_{q} \in \mathscr{C}$ and $v_{q} \neq 0$,

$$
\begin{aligned}
\widehat{\mathrm{T}_{v_{q}} \mathscr{C}} & :=\mathrm{T}_{v_{q}} \mathscr{C} /\left[X_{\mathscr{C}}\left(R_{\mathcal{F}}\right)\left(v_{q}\right)\right] \\
& \equiv\left\{X_{v_{q}} \in \mathrm{T}_{v_{q}} \mathscr{C} \mid\left\langle\mathrm{T} \pi_{\mathscr{C}} \cdot X_{v_{q}}, v_{q}\right\rangle=0\right\} .
\end{aligned}
$$


Indeed, it suffices to show that for all $v_{q} \in \mathscr{C}$ and $v_{q} \neq 0$,

$\left\langle\mathrm{T} \pi_{\mathscr{C}} \cdot X_{\mathscr{C}}\left(R_{\mathcal{F}}\right)\left(v_{q}\right), v_{q}\right\rangle \neq 0$. But $X_{\mathscr{C}}\left(R_{\mathcal{F}}\right)\left(v_{q}\right)=\mathrm{S}\left(v_{q}\right)+\lambda_{v_{q}}\left(\mathcal{F}^{\sharp}\left(v_{q}\right)+\right.$ $R_{\mathcal{F}}\left(v_{q}\right)$ ), where $\mathrm{S}$ is the geodesic spray of the Levi-Civita connection associated to $(\mathrm{M}, \mathrm{g})$, so that $\left\langle\mathrm{T} \pi_{\mathscr{C}} \cdot X_{\mathscr{C}}\left(R_{\mathcal{F}}\right)\left(v_{q}\right), v_{q}\right\rangle=\left\langle v_{q}, v_{q}\right\rangle>0$, since $v_{q} \neq 0$.

Define $H: \mathrm{T} \mathscr{C} \rightarrow \mathbb{R}$ as $H\left(X_{v_{q}}\right):=\frac{1}{2}\left\|P_{\left[v_{q}\right]^{\perp}} \cdot \mathrm{T} \pi_{\mathscr{C}} \cdot X_{v_{q}}\right\|^{2}$, where $P_{\left[v_{q}\right]^{\perp}}$ is the orthogonal projection. Then, for all $v_{q} \in \mathscr{C}, X_{v_{q}} \in \mathrm{T}_{v_{q}} \mathscr{C}, s \in \mathbb{R}$, we have $H\left(X_{v_{q}}+s X_{\mathscr{C}}\left(R_{\mathcal{F}}\right)\right)=H\left(X_{v_{q}}\right)$, so that $H$ passes to the quotient $\widehat{\mathrm{T} \mathscr{C}}$. Let $Q:=\mathfrak{L}_{X_{\mathscr{C}}\left(R_{\mathcal{F}}\right)} H$, so that $Q$ also passes to the quotient $\widehat{\mathrm{T} \mathscr{C}}$. Applying Lemma 1 we obtain, after a direct computation:

$$
\begin{aligned}
Q\left(X_{v_{q}}\right)=\frac{d}{d t}_{t_{t=0}} H\left(\mathrm{~T} \phi^{t} \cdot X_{v_{q}}\right)= \\
\quad=\left\langle\mathrm{T} \pi_{\mathscr{C}} \cdot X_{v_{q}}, \kappa \cdot X_{v_{q}}\right\rangle \\
\quad-\frac{\left\langle\mathrm{T} \pi_{\mathscr{C}} \cdot X_{v_{q}}, v_{q}\right\rangle}{\left\langle v_{q}, v_{q}\right\rangle}\left(\left\langle\kappa \cdot X_{v_{q}}, v_{q}\right\rangle+\left\langle\mathrm{T} \pi_{\mathscr{C}} \cdot X_{v_{q}}, \mathcal{F}^{\sharp}\left(v_{q}\right)+R_{\mathcal{F}}\left(v_{q}\right)\right\rangle\right) \\
\quad+\frac{\left\langle\mathrm{T} \pi_{\mathscr{C}} \cdot X_{v_{q}}, v_{q}\right\rangle^{2}}{\left\langle v_{q}, v_{q}\right\rangle^{2}}\left\langle v_{q}, \mathcal{F}^{\sharp}\left(v_{q}\right)+R_{\mathcal{F}}\left(v_{q}\right)\right\rangle
\end{aligned}
$$

In $\widehat{\mathrm{T} \mathscr{C}}$ putting $\left\langle\mathrm{T} \pi_{\mathscr{C}} \cdot X_{v_{q}}, v_{q}\right\rangle=0$ in the right hand side of the previous equation we obtain $\hat{Q}\left(X_{v_{q}}\right)=Q_{1}\left(v_{q}\right)\left(X_{v_{q}}\right)$.

Computing the Lie derivative $\mathfrak{L}_{X_{\mathscr{C}}\left(R_{\mathcal{F}}\right)} Q$ using Lemma 1 and again putting $\left\langle\mathrm{T} \pi_{\mathscr{C}} \cdot X_{v_{q}}, v_{q}\right\rangle=0$ to pass to the quotient $\widehat{\mathrm{T} \mathscr{C}}$, we obtain $\widehat{\mathfrak{L}_{X_{\mathscr{C}}\left(R_{\mathcal{F}}\right)}} Q\left(X_{v_{q}}\right)=Q_{2}\left(v_{q}\right)\left(X_{v_{q}}\right)$. So the hypotheses of the proposition guarantees that the flow $\left(\phi^{t}\right)_{t \in \mathbb{R}}$ is strictly monotonous and thus hyperbolic.

Example 1. Let $(\mathrm{M}, \mathrm{g})$ be a compact, Riemannian manifold and consider in $\mathrm{M}$ the isokinetic constraint given by $\mathscr{C}=\left\{v_{q} \in \mathrm{TM} \mid\left\langle v_{q}, v_{q}\right\rangle=e^{2}\right\}, e \neq 0$ (see [16], [35], [13] and [42]). Consider also a magnetic field $\mathbf{B} \in \Omega_{2}(\mathbf{M})$, with the corresponding Lorentz force $\mathscr{Y}: \mathrm{TM} \rightarrow \mathrm{TM}$

$$
\left(\forall q \in \mathrm{M}, v_{q}, w_{q} \in \mathrm{T}_{q} \mathrm{M}\right)\left\langle v_{q}, \mathscr{Y}\left(w_{q}\right)\right\rangle=\mathbf{B}\left(v_{q}, w_{q}\right),
$$

and a potential $V \in \mathfrak{F}(\mathrm{M})$. Let $\mathcal{F}^{\sharp}:=\mathscr{Y}-\operatorname{grad} V \circ \tau_{\mathrm{M}}$. We will obtain a condition such that the flow of the GMA vector field $X_{\mathscr{C}}$ is hyperbolic on $\mathscr{M}=\mathscr{C}$. 
Given $v_{q} \in \mathscr{C}$, we have $C_{v_{q}}=\left[v_{q}\right]^{\perp}, W_{v_{q}}=\lambda_{v_{q}}\left[v_{q}\right]$. Therefore, $\mathrm{T}_{v_{q}} \mathscr{C}=\left\{X_{v_{q}} \in \mathrm{T}_{v_{q}} \mathrm{TM} \mid\left\langle\kappa \cdot X_{v_{q}}, v_{q}\right\rangle=\left\langle\mathrm{T} \pi_{\mathscr{C}} \cdot X_{v_{q}}, v_{q}\right\rangle=0\right\}$, and by polarization $Q_{1}$ is non-degenerate. Moreover, writing $\xi_{q}:=\mathrm{T} \pi_{\mathscr{C}} \cdot X_{v_{q}}$, $\eta_{q}:=\kappa \cdot X_{v_{q}}$, a simple computation shows that:

$$
\begin{aligned}
Q_{2}\left(v_{q}\right) & \left(X_{v_{q}}\right)=\left\|\eta_{q}-\frac{\mathscr{Y}\left(\xi_{q}\right)}{2}-\frac{1}{2 e^{2}}\left\langle\operatorname{grad} V(q), v_{q}\right\rangle \xi_{q}\right\|^{2} \\
& -\frac{\left\|\mathscr{Y}\left(\xi_{q}\right)\right\|^{2}}{4}-\frac{1}{4 e^{4}}\left\|\left\langle\operatorname{grad} V(q), v_{q}\right\rangle \xi_{q}\right\|^{2}+\left\langle\mathrm{R}\left(v_{q}, \xi_{q}\right) \cdot v_{q}, \xi_{q}\right\rangle \\
& -\left\langle\nabla_{\xi_{q}} \operatorname{grad} V, \xi_{q}\right\rangle+\left\langle\left(\nabla_{\xi_{q}} \mathscr{Y}\right)\left(v_{q}\right), \xi_{q}\right\rangle \\
& -\frac{1}{e^{4}}\left\langle\xi_{q},-\operatorname{grad} V(q)+\mathscr{Y}\left(v_{q}\right)\right\rangle^{2} .
\end{aligned}
$$

Hence, if for all $v_{q} \in \mathscr{C}$ the quadratic form:

$$
\begin{aligned}
\xi_{q} \mapsto & -\frac{\left\|\mathscr{Y}\left(\xi_{q}\right)\right\|^{2}}{4}-\frac{1}{4 e^{4}}\left\|\left\langle\operatorname{grad} V(q), v_{q}\right\rangle \xi_{q}\right\|^{2}+\left\langle\mathrm{R}\left(v_{q}, \xi_{q}\right) \cdot v_{q}, \xi_{q}\right\rangle \\
& -\left\langle\nabla_{\xi_{q}} \operatorname{grad} V, \xi_{q}\right\rangle+\left\langle\left(\nabla_{\xi_{q}} \mathscr{Y}\right)\left(v_{q}\right), \xi_{q}\right\rangle \\
& -\frac{1}{e^{4}}\left\langle\xi_{q},-\operatorname{grad} V(q)+\mathscr{Y}\left(v_{q}\right)\right\rangle^{2}
\end{aligned}
$$

is positive definite in $\left[v_{q}\right]^{\perp} \subset \mathrm{T}_{q} \mathrm{M}$, then the flow of the GMA vector field $X_{\mathscr{C}}$ is hyperbolic. This result agrees with the quadratic form in theorem 4.1 of [42] and, for example, if the Riemannian manifold ( $M, g$ ) has a sectional curvature smaller than $-k^{2}<0$ and if $\mathbf{B}$ and $V$ are such that:

$$
\frac{\|\nabla \mathscr{Y}\|}{e}+\frac{\|\nabla \operatorname{grad} V\|}{e^{2}}+\left(\frac{\|\mathscr{Y}\|}{e}+\frac{\operatorname{grad} V}{e^{2}}\right)^{2}<k^{2},
$$

then the flow of $X_{\mathscr{C}}$ is hyperbolic. Note that in the particular case of $\mathbf{B} \equiv 0$ and $V \equiv 0$, we recover the well known result of Anosov, which asserts that the geodesic flow in the unitary tangent bundle of a Riemannian manifold with negative sectional curvature is hyperbolic.

\section{A GMA invariant measure}

In [21] and [22] it is considered the question of the conservation of Riemannian volume in a series of classical examples of non-holonomic mechanical systems with linear as well as non-linear constraints. General necessary and sufficient conditions for the conservation of volume for both latter cases have been reported in [27] and [36] (see also [43]). 
Given a constrained mechanical system $(\mathrm{M}, \mathrm{K}, \mathcal{F}, \mathscr{C})$, let $\mu$ be the Radon measure induced on $\mathscr{C}$ by the Sasaki metric $\mathrm{g}_{\mathscr{C}}$ (see definition 3 on page 61 ). We present a necessary and sufficient condition for the existence of a GMA invariant measure of the form $\left(f \circ \pi_{\mathscr{C}}\right) \mu$, where $f$ is a smooth positive function on $\mathrm{M}$. This is a generalization of a similar condition stated in [5] for linearly constrained systems.

We recall the following definition from [36]:

Definition 9. We denote by $A: \mathscr{C} \rightarrow \mathrm{L}(\mathrm{TM}, \mathrm{TM})$ the smooth map defined by, for all $v_{q} \in \mathscr{C}, A\left(v_{q}\right):=\kappa \circ P_{\mathscr{C}} \circ \mathrm{H}_{v_{q}}: \mathrm{T}_{q} \mathrm{M} \rightarrow \mathrm{T}_{q} \mathrm{M}$, where $\kappa$ is the connector induced by the Levi-Civita connection of (M,g).

Remark 2. For a linear constraint $\mathscr{D}$, a direct computation shows that the map $A$ of the previous definition is given by, for all $v_{q} \in \mathscr{D}$, $A\left(v_{q}\right)=B_{\mathscr{D}}\left(v_{q}\right)$, where $B_{\mathscr{D}}: \mathrm{TM} \oplus_{\mathrm{M}} \mathscr{D} \rightarrow \mathscr{D}^{\perp}$ is the total second fundamental form of $(\mathrm{M}, \mathrm{g}, \mathscr{D})$ - see $[27]$ - and $B_{\mathscr{D}}\left(v_{q}\right)=B_{\mathscr{D}}\left(\cdot, v_{q}\right): \mathrm{T}_{q} \mathrm{M} \rightarrow \mathscr{D}_{q}^{\perp}$. In this sense, the map $A$ of the previous definition plays the role, in the non-linearly constrained case, of the total second fundamental form.

Notation. Given $q \in \mathrm{M}, v_{q} \in \mathscr{C}_{q}$ and $w_{q} \in \mathrm{T}_{q} \mathrm{M}$, we denote by $\mathbb{F}^{*} \mathscr{P}\left(v_{q}\right) \cdot w_{q}$ the adjoint map of $\mathbb{F} \mathscr{P}\left(v_{q}\right) \cdot w_{q}: \mathrm{T}_{q} \mathrm{M} \rightarrow \mathrm{T}_{q} \mathrm{M}$ with respect to the metric tensor. This defines the map: $\mathbb{F}^{*} \mathscr{P}: \mathscr{C} \rightarrow \mathrm{L}(\mathrm{TM}, \mathrm{L}(\mathrm{TM}, \mathrm{TM})) \equiv \mathrm{L}(\mathrm{TM} \otimes$ TM, TM).

We can now state the following:

THEOREM B. With the definition and notation stated above, let $\mu$ be the Radon measure induced on $\mathscr{C}$ by the Sasaki metric $\mathrm{g}_{\mathscr{C}}$. The existence of a GMA invariant measure of the form $\left(f \circ \pi_{\mathscr{C}}\right) \mu$, where $f$ is a smooth positive function on $\mathrm{M}$, is equivalent to the existence of an exact 1-form $\theta$ on $\mathrm{M}$ such that the restriction to $\mathscr{C}$ of the smooth function $\widehat{\theta}: \mathrm{TM} \rightarrow \mathbb{R}$ induced by $\theta$ coincides with $v_{q} \mapsto-\operatorname{tr} A\left(v_{q}\right)-\left\langle\left.\operatorname{tr} \mathbb{F}^{*} \mathscr{P}\left(v_{q}\right)\right|_{C_{v_{q}} \times C_{v_{q}}}, R_{\mathcal{F}}^{A}\left(v_{q}\right)\right\rangle$, where $R_{\mathcal{F}}^{A}$ is given by (8).

Proof. Let $\omega$ be the Riemannian volume induced on $\mathscr{C}$ (locally defined if $\mathscr{C}$ is not orientable) by the Sasaki metric tensor. The Radon measure $\mu$ is then induced by the volume form $\omega$ and, for a given smooth positive function $f$ on $\mathrm{M}$, the Radon measure $\left(f \circ \pi_{\mathscr{C}}\right) \mu$ is induced by the volume form $\Omega \doteq\left(f \circ \pi_{\mathscr{C}}\right) \omega$. Since $\mathfrak{L}_{X_{\mathscr{C}}}\left[\left(f \circ \pi_{\mathscr{C}}\right) \omega\right]=X_{\mathscr{C}}\left[f \circ \pi_{\mathscr{C}}\right] \omega+\left(f \circ \pi_{\mathscr{C}}\right) \mathfrak{L}_{X_{\mathscr{C}}} \omega$, it follows that the divergences of the GMA vector field $X_{\mathscr{C}}$ with respect to these volume forms are related by $\operatorname{div}_{\Omega} X_{\mathscr{C}}=\frac{X_{\mathscr{C}}\left[f \circ \pi_{\mathscr{C}}\right]}{f \circ \pi_{\mathscr{C}}}+\operatorname{div}_{\omega} X_{\mathscr{C}}$. But, as $\frac{X_{\mathscr{C}}\left[f \circ \pi_{\mathscr{C}}\right]}{f \circ \pi_{\mathscr{C}}}=X_{\mathscr{C}}\left[\log \circ f \circ \pi_{\mathscr{C}}\right]$ and $X_{\mathscr{C}}$ is a second order vector field (i.e. 
$\left.\mathrm{T} \pi_{\mathscr{C}} \circ X_{\mathscr{C}}=\mathrm{id} \mathscr{C}_{\mathscr{C}}\right)$, we have, for all $v_{q} \in \mathscr{C}, \frac{X_{\mathscr{C}}\left[f \circ \pi_{\mathscr{C}}\right]}{f \circ \pi_{\mathscr{C}}}\left(v_{q}\right)=\mathrm{d}(\log \circ f) \cdot \mathrm{T} \pi_{\mathscr{C}}$. $X_{\mathscr{C}}\left(v_{q}\right)=\mathrm{d}(\log \circ f) \cdot v_{q}$. Hence, for all $v_{q} \in \mathscr{C}$ :

$$
\operatorname{div} \Omega X_{\mathscr{C}}\left(v_{q}\right)=\mathrm{d}(\log \circ f) \cdot v_{q}+\operatorname{div}_{\omega} X_{\mathscr{C}}\left(v_{q}\right)
$$

From the equation above we conclude that, if the Radon measure $(f \circ$ $\left.\pi_{\mathscr{C}}\right) \mu$ is invariant under the flow of $X_{\mathscr{C}}$ (i.e. if $\operatorname{div} \Omega X_{\mathscr{C}}$ vanishes on $\mathscr{C}$ ), the function $\mathrm{TM} \rightarrow \mathbb{R}$ induced by the exact 1 -form $\theta \doteq \mathrm{d}(\log \circ f)$ coincides on $\mathscr{C}$ with $-\operatorname{div}_{\omega} X_{\mathscr{C}}$. Conversely, if there exists an exact 1 -form $\theta=\mathrm{d} g$ on $\mathrm{M}$ satisfying the last condition, we take $f \doteq \exp g$, and then $\operatorname{div} \Omega X_{\mathscr{C}}$ vanishes on $\mathscr{C}$, by equation (10). Since, for all $v_{q} \in \mathscr{C}, \operatorname{div}_{\omega} X_{\mathscr{C}}\left(v_{q}\right)=$ $\operatorname{tr} A\left(v_{q}\right)+\left\langle\left.\operatorname{tr} \mathbb{F}^{*} \mathscr{P}\left(v_{q}\right)\right|_{C_{v_{q}} \times C_{v_{q}}}, R_{\mathcal{F}}^{A}\left(v_{q}\right)\right\rangle$, as it is computed in [36], the thesis follows.

Example 2. We now apply theorem B to show that the GMA flow of a constrained mechanical system $(\mathrm{M}, \mathrm{K}, \mathcal{F}, \mathscr{C})$ where

$\mathscr{C}=\left\{v_{q} \in \mathrm{TM} \mid\left\langle v_{q}, v_{q}\right\rangle=1\right\}$, the isokinetic dynamics (see references in example 1), admits an invariant measure if the force field derives from a potential, i.e. if $\mathcal{F}=\mathrm{dV}$ for some smooth function $\mathrm{V}$ on $\mathrm{M}$ (or, more generally, if $\mathcal{F}$ coincides on $\mathscr{C}$ with $\mathrm{dV}$ ).

Using the notation fixed in section 2.2 , we have $C_{v_{q}}=\left[v_{q}\right]^{\perp}$ and $W_{v_{q}}=\lambda_{v_{q}}\left[v_{q}\right]$. Then $\mathscr{P}: \mathscr{C} \rightarrow \mathrm{L}(\mathrm{TM}, \mathrm{TM})$ is given by, for all $v_{q} \in \mathscr{C}$, $\mathscr{P}\left(v_{q}\right): w_{q} \mapsto w_{q}-\left\langle w_{q}, v_{q}\right\rangle v_{q}$, and $P_{W}: i_{\mathscr{C}}^{*}(\mathrm{TTM}) \rightarrow W$ is given by $X_{v_{q}} \mapsto \lambda_{v_{q}}\left(\left\langle\kappa \cdot X_{v_{q}}, v_{q}\right\rangle v_{q}\right)$. It then follows that, for all $v_{q} \in \mathscr{C}$, $A\left(v_{q}\right)=\kappa \cdot P_{\mathscr{C}} \cdot \mathrm{H}_{v_{q}}=-\kappa \cdot P_{W} \cdot \mathrm{H}_{v_{q}}=0$, i.e. $A \equiv 0$. Besides, a direct computation shows that, for all $v_{q} \in \mathscr{C}$ and all $w_{q} \in C_{v_{q}}$, $\mathbb{F} \mathscr{P}\left(v_{q}\right)\left(w_{q}, R_{\mathcal{F}}^{A}\left(v_{q}\right)\right)=-\left\langle\mathcal{F}\left(v_{q}\right), v_{q}\right\rangle w_{q}$. Thus $\left(\forall v_{q} \in \mathscr{C}, \forall w_{q}, z_{q} \in C_{v_{q}}\right)\left\langle\mathbb{F}^{*} \mathscr{P}\left(v_{q}\right)\left(w_{q}, z_{q}\right), R_{\mathcal{F}}^{A}\left(v_{q}\right)\right\rangle=-\left\langle z_{q}, w_{q}\right\rangle\left\langle\mathcal{F}\left(v_{q}\right), v_{q}\right\rangle$. Hence, for all $v_{q} \in \mathscr{C}$ :

$$
\begin{aligned}
-\operatorname{tr} A\left(v_{q}\right)-\left\langle\left.\operatorname{tr} \mathbb{F}^{*} \mathscr{P}\left(v_{q}\right)\right|_{C_{v_{q}} \times C_{v_{q}}}, R_{\mathcal{F}}^{A}\left(v_{q}\right)\right\rangle & =\left.\operatorname{tr} \mathrm{g}\right|_{C_{v_{q}} \times C_{v_{q}}}\left\langle\mathcal{F}\left(v_{q}\right), v_{q}\right\rangle= \\
& =(n-1) \mathrm{dV}(q) \cdot v_{q}
\end{aligned}
$$

where $n=\operatorname{dim} \mathrm{M}$. By theorem B, we conclude that the GMA flow admits an invariant measure. Explicitly, the measure $\exp [(n-1) \mathrm{V}] \mu$, where $\mu$ is the Radon measure induced by the Sasaki metric tensor on $\mathscr{C}$, is invariant under the flow of $X_{\mathscr{C}}$.

Example 3. For a linearly constrained mechanical system $(\mathrm{M}, \mathrm{K}, \mathcal{F}, \mathscr{D})$, we have $\mathbb{F} \mathscr{P} \equiv 0$ and $\left(\forall v_{q} \in \mathscr{C}\right) A\left(v_{q}\right)=B_{\mathscr{D}}\left(v_{q}\right)=B_{\mathscr{D}}\left(\cdot, v_{q}\right): \mathrm{T}_{q} \mathrm{M} \rightarrow \mathscr{D}_{q}^{\perp}$. Thus $\left(\forall v_{q} \in \mathscr{C}\right)-\operatorname{tr} A\left(v_{q}\right)-\left\langle\left.\operatorname{tr} \mathbb{F}^{*} \mathscr{P}\left(v_{q}\right)\right|_{C_{v_{q}} \times C_{v_{q}}}, R_{\mathcal{F}}^{A}\left(v_{q}\right)\right\rangle=-\operatorname{tr} B_{\mathscr{D}}\left(v_{q}\right)=$ $-\left\langle\left.\operatorname{tr} B_{\mathscr{D} \perp}\right|_{\mathscr{D} \frac{\perp}{q} \times \mathscr{Q}} ^{\perp}, v_{q}\right\rangle$. It then follows that the condition stated in theorem $\mathrm{B}$ for the existence of a GMA invariant measure is equivalent to the section 
of $\mathscr{D}^{*}$ given by $q \in \mathrm{M} \mapsto\left\langle\left.\operatorname{tr} B_{\mathscr{D} \perp}\right|_{\mathscr{D}_{q}^{\perp} \times \mathscr{D}}, \cdot\right\rangle \in \mathscr{D}_{q}^{*}$ being extendable to an exact 1-form on M. ${ }^{4}$ Note that this condition is independent of the force field $\mathcal{F}$. This is a slight improvement on Blackall's condition for the existence of such an invariant measure (see [5]).

\section{Conservation of volume: the rolling of a ball on a surface parallel to Delaunay}

In the linear case, that is, when the constraint is a vector sub-bundle $\mathscr{D} \subset$ TM of the tangent bundle TM, the conservation of the Riemannian volume is equivalent to the trace of the restriction of the total second fundamental form to the sub-bundle $\mathscr{D}^{\perp} \subset \mathrm{TM}$ being null; in particular (see Corollary 3 of [21]) that condition together with the integrability of the distribution $\mathscr{D}^{\perp}$ imply that $\mathscr{D}^{\perp}$ defines a foliation on $\mathrm{M}$ with minimal leaves.

One of the most significant examples is given by the non-slipping rolling of a homogeneous ball of radius $\delta>0$ over an embedded surface $S \subset \mathbb{R}^{3}$. For this kind of non-holonomic mechanical system with linear constraint $\mathscr{D}$, its GMA flow always conserves the Riemannian volume (see Theorem 6 of $[22]$ ) induced on $\mathscr{D}$ (see [27], [36]). Moreover (see Theorem 6 [22]), in that case the distribution $\mathscr{D}^{\perp}$ is integrable (involutive) if and only if the surface parallel to $S$, defined as the set $C$ of all centers of all possible positions of the ball, is a surface of constant mean curvature with modulus equal to $5 / 4 \delta$ (for the definition of parallel surface see [32]). Since Delaunay surfaces represent all the surfaces of revolution embedded in $\mathbb{R}^{3}$ with constant mean curvature (with the exception of spheres) the question one needs to analyze now is the following: what is the surface $S$ such that its "parallel" surface $C$ is a Delaunay surface?

In order to answer that last question we start by recalling some properties of Delaunay surfaces.

In 1841 Delaunay [10] was able to obtain the following description of all surfaces of revolution in $\mathbb{R}^{3}$ of constant mean curvature (see also [17]). By rolling a given conic section on a line in a plane, and rotating about that line the trace of a focus, one obtains a surface of constant mean curvature in $\mathbb{R}^{3}$. Conversely, all the surfaces of revolution of constant mean curvature in $\mathbb{R}^{3}$ (with the exception of spheres) can be described in this way:

Theorem (Delaunay [10]). For finding the meridian curve of a surface of revolution of which the mean curvature is constant and equal to $(2 a)^{-1}$,

4. For instance, if the first Betti number of $M$ is null, it is sufficient for the existence of a GMA invariant measure that $\mathrm{d}\left[\left.\operatorname{tr} B_{\mathscr{D}}\right|_{\mathscr{D}} \times_{\times \mathscr{D}}\right]^{\mathrm{b}}=0$.

São Paulo J.Math.Sci. 2, 1 (2008), 55-76 
it must be done by rolling upon the axis of the surface an ellipse (or a hyperbola) such that the major axis (or the transverse axis) is equal to $2 a$, and the focus describes the desired curve.

The limiting case, that is, if is equal to zero the mean curvature of a surface of revolution of which one seeks the meridian curve, then this curve will be generated by the trace of the focus of a parabola rolling upon the axis of the surface. One knows that this curve is the catenary and the surface of revolution is the catenoid, a minimal surface of $\mathbb{R}^{3}$.

Delaunay obtained also parametric equations for the surface in terms of positively oriented orthogonal coordinates $(x, y)$ on the plane of the meridian curve, the $\mathrm{x}$ coordinates giving the orientation of the axis of rotation of the surface. For the proof he started by integrating the equations for an evolute of the meridian curve and from that he obtained the parametric equations. Therefore these last equations hold only on some intervals on which the evolute can be defined.

Kenmotsu [19] found nice expressions for the meridian curve in terms of the arc length parameter and described, among other interesting results, all complete surfaces of revolution in $\mathbb{R}^{3}$ with constant mean curvature. In fact, Kenmotsu studied the surfaces of revolution in $\mathbb{R}^{3}$ with prescribed mean curvature $H(s)$ where $s$ is the arc length of the $C^{2}$ meridian curve $(x(s), y(s)), s \in I$. The surface of revolution generated by the rotation of that meridian curve, around the $x$-axis, have the following representation:

$$
(x(s), y(s) \cos \theta, y(s) \sin \theta), \quad s \in I \quad \theta \in[0,2 \pi] .
$$

By the regularity of the surface, we may assume $y(s)>0$ on the interval $I$.

So the mean curvature $H(s)$, satisfies,

$$
2 H(s) y(s)-x^{\prime}(s)-y(s)\left[x^{\prime \prime}(s) y^{\prime}(s)-x^{\prime}(s) y^{\prime \prime}(s)\right]=0
$$

where $x^{\prime}(s)^{2}+y^{\prime}(s)^{2}=1, \quad s \in I$.

If the mean curvature is constant and non zero, $H(s)=H \neq 0$, Kenmotsu derived the following expression for the meridian curve $(x(s), y(s))=X(s ; H, B)$, where $B$ is any constant (see [19]):

$$
\begin{aligned}
& X(s ; H, B)= \\
& \quad=\left(\int_{0}^{s} \frac{1+B \sin 2 H t}{\sqrt{1+B^{2}+2 B \sin 2 H t}} d t, \frac{1}{2|H|} \sqrt{1+B^{2}+2 B \sin 2 H s}\right),
\end{aligned}
$$

$s \in \mathbb{R}$.

The following interesting remarks are useful (see [19]):

(a) $X(s ;-H, B)=X(s ; H,-B)$;

(b) $X(s ; H,-B)=X\left(s-\frac{\pi}{2} H ; H, B\right)+$ a constant vector; 
(c) $X(s ; \lambda H, B)=\frac{1}{\lambda} X(\lambda s ; H, B), \lambda>0$.

So, without loss of generality, it is enough to consider the cases $B \geq 0$ and $H>0$.

(d) $X(s ; H, 0)$ is the generating line of a circular cylinder;

(e) $X(s ; H, 1)$ represents a sequence of continuous half circles over the $x$-axis which have the radii;

(f) If $0<B<1, x(s)$ is monotone increasing as $s \rightarrow \infty$.

(g) If $B>1 x(s)$ is not monotone.

In the last two cases, $\lim x(s)=\infty$ as $s \rightarrow \infty$ because $X(s ; H, B)$ is periodic, with period $\pi / H$. Moreover, using Delaunay's Theorem 5 a simple computation shows that the constants $H$ and $B$ appearing in (12) are given by $H=1 / 2 a$ and $B=e$, where $a$ and $e$ are the semi-axis and the eccentricity of the rolling ellipse or hyperbola. Note that in the case $B>1$ we obtain points where $x^{\prime}(s)$ vanishes, that is, the values of $s \in \mathbb{R}$ such that $\sin 2 H s=-\frac{1}{B}$.

From (11) one obtains

$$
2 H=\frac{x^{\prime}}{y}+x^{\prime \prime} y^{\prime}-x^{\prime} y^{\prime \prime}=\frac{1}{a} .
$$

Since $\tau=\left(x^{\prime}, y^{\prime}\right), n=\left(-y^{\prime}, x^{\prime}\right)$ for $(\tau, n)$ positively oriented and $\tau^{\prime}=k_{c} n$ (see [11]) we have $x^{\prime \prime} y^{\prime}-x^{\prime} y^{\prime \prime}=-k_{c}$. And so we can rewrite (11) as

$$
k_{p}+k_{m}=\frac{1}{a}
$$

where $k_{p}$ and $k_{m}$ are the principal curvatures

$$
k_{p}=\frac{x^{\prime}}{y}
$$

and

$$
k_{m}=-k_{c}=x^{\prime \prime} y^{\prime}-x^{\prime} y^{\prime \prime} .
$$

Remark 3. Standard computations give us the following expressions

$$
k_{p}=\frac{y^{2} \pm b^{2}}{2 a y^{2}}
$$

and

$$
k_{m}=\frac{y^{2} \mp b^{2}}{2 a y^{2}}
$$

where the upper (resp. the lower) sign corresponds to the ellipse of equation $\frac{x^{2}}{a^{2}}+\frac{y^{2}}{b^{2}}=1$ (resp. hyperbola of equation $\left.\frac{x^{2}}{a^{2}}-\frac{y^{2}}{b^{2}}=1\right)$. 
We will show that the surface $S \subset \mathbb{R}^{3}$ where the sphere rolls is well defined, globally, as an embedding. The surface $S$ is parallel to the given Delaunay surface $C \subset \mathbb{R}^{3}$ defined by the locus of the positions of the center of the sphere when it rolls over $S$. We consider two types of Delaunay surfaces. For $0<B<1$ the meridian curve generating the Delaunay surface is periodic and $x^{\prime}>0$. In this case, there is no "loop" in the meridian and we consider $S_{ \pm}$generated by the meridian curve extending from $x \rightarrow-\infty$ to $x \rightarrow+\infty$. When $B>1$, the meridian is still periodic, but the curve extending from $x \rightarrow-\infty$ to $x \rightarrow+\infty$ presents loops. So to avoid these loops, we consider in the sequel the Delaunay surface generated by rotating the generating curve $(x(s), y(s))$ above over one period and starting at any point where $x^{\prime}=0$.

Let us now choose the field of unit normal vectors $\xi$ on the surface $C$ such that at each point $\xi$ coincides with $-n$, where $n$ is the above unit normal to the meridian at that point.

Define $S_{+}$(resp. $S_{-}$), the parallel surface to $C$, by $S_{+}=C+\delta \xi$ (resp. $S_{-}=C-\delta \xi$ ), with $\delta$ to be chosen properly. The condition for $S_{+}$to be an immersion in $\mathbb{R}^{3}$ is that the parallel distance between $S_{+}$and $C$, given by the radius of the sphere, be distinct from the reciprocal of the curvature $k_{c}$ of the meridian generating $C$. To show that, let us denote by $(\bar{x}, \bar{y})$ a generic point of the meridian of $S_{+}$. So

$$
(\bar{x}, \bar{y})=(x, y)+\delta\left(\xi_{x}, \xi_{y}\right),
$$

and

$$
\left(\bar{x}^{\prime}, \bar{y}^{\prime}\right)=\left(x^{\prime}, y^{\prime}\right)+\delta\left(\xi_{x}^{\prime}, \xi_{y}^{\prime}\right),
$$

where / denotes the derivation with respect to the arc length of the meridian curve of the surface $C$. From what we saw above one has

$$
\xi^{\prime}=k_{c}\left(x^{\prime}, y^{\prime}\right)
$$

then

$$
\left(\bar{x}^{\prime}, \bar{y}^{\prime}\right)=\left(x^{\prime}+\delta k_{c} x^{\prime}, y^{\prime}+\delta k_{c} y^{\prime}\right)=\left(1+\delta k_{c}\right)\left(x^{\prime}, y^{\prime}\right) .
$$

So we have immersion if and only if $1+\delta k_{c} \neq 0$ along the meridian curve (in fact, we only need to check the previous condition for points where $\left.k_{c}<0\right)$. For $S_{-}$the condition for immersion is $1-\delta k_{c} \neq 0$ and is obtained analogously.

We will prove that the same condition obtained for the immersion is enough to show that we obtain the embedding of the surfaces $S_{+}$and $S_{-}$. Start by fixing $\tilde{s} \in \mathbb{R}$ and consider the map $f: s \mapsto \bar{x}(s)-\bar{x}(\tilde{s}) \in \mathbb{R}$. We need to show the injectivity of the function $(x, y) \in C \mapsto(\bar{x}, \bar{y}) \in S_{+}$and for that it is enough to show that for any $s \neq \tilde{s}$ we have $\bar{x}(s) \neq \bar{x}(\tilde{s})$. Now assume by contradiction that there exists an $\hat{s} \neq \tilde{s}$ such that $\bar{x}(\hat{s})=\bar{x}(\tilde{s})$, so we have $f(\hat{s})=0$ and also $f(\tilde{s})=0$. By the classical Rolle's theorem 
there exists $s_{0}$ strictly between $\hat{s}$ and $\tilde{s}$ such that $f^{\prime}\left(s_{0}\right)=x^{\prime}\left(1-\delta k_{c}\right)=0$, with $\delta k_{c} \neq 1$, which is a contradiction because the meridian curve of the Delaunay surface does not have vertical tangents (the same happens with $\left.S_{-}\right)$. That proves the following proposition:

Proposition 2. Let $k_{c}$ be the curvature function of the meridian curve of the Delaunay surface and $\delta>0$ such that $k_{c} \delta \neq 1$ (resp. $\left.k_{c} \delta \neq-1\right)$. Then $S_{+}$(resp. $\left.S_{-}\right)$is an embedded parallel surface to a given Delaunay surface.

The existence of a "free" rolling motion of a ball of radius $\delta>0$ over the surface $S_{+}$(resp. $S_{-}$) requires that the sphere is not "locked" during its motion over that surface. Here not locked means that the reciprocal of the radius $\delta$ of the ball is bigger than the maximum of the absolute values of the principal curvatures of the surface $S_{+}$(resp. $S_{-}$) for all points where the ball touches the closure of the set of points of the meridian of the surface $S_{+}$(resp. $S_{-}$) in which this meridian is convex.

Using the previous definitions and results, and using the relation between the principal curvatures of the Delaunay surface $C$ and its parallel surfaces $S_{ \pm}($see $[32])$

$$
k_{i}^{S_{ \pm}}=\frac{k_{i}^{C}}{1 \mp \delta k_{i}^{C}}
$$

valid for $\delta<\left|k_{i}^{C}\right|$; a simple computation then shows:

Theorem C. The surface $S_{+}$(resp. $S_{-}$) parallel to a given Delaunay surface $C$, as defined above, is well defined and the ball can freely roll over it, if the appropriate condition in the table below is satisfied.

TABLE 1. Conditions for the sphere to roll over a surface parallel to a Delaunay surface.

\begin{tabular}{lcc}
\hline & Ellipse & Hyperbola \\
\hline$S_{-}$ & $\delta< \begin{cases}a & \text { if } 0<e<1 / 2 \\
\frac{a(1-e)}{e} & \text { if } e \geq 1 / 2\end{cases}$ & $\delta<a(e-1)$ \\
$\delta<a(1-e)$ & $\delta<\frac{a(e-1)}{e}$ \\
\hline
\end{tabular}

\section{References}

[1] R. Abraham and J. E. Marsden, Foundations of Mechanics, Addison-Wesley Publishing Company, Reading, Massachusetts, second ed., 1978.

[2] P. Appell, Traité de Mécanique Rationelle-Tome II, Dynamique des systèmes. - Mécanique analytique, Gauthier-Villars, Paris, sixth ed., 1941. 
[3] V. I. Arnold, V. V. Kozlov, And A. I. Neishtadt, Dynamical Systems III, vol. 3 of Encyclopaedia of Mathematical Sciences, Springer-Verlag, New York, 1988, ch. Mathematical Aspects of Classical and Celestial Mechanics, pp. 1-286.

[4] S. BENENTI, Geometrical aspects of the dynamics of non-holonomic systems, Rendiconti del Seminario Matematico dell' Univivesitá e del Politecnico di Torino, 54 (1996), pp. 203-212.

[5] C. BlackALL, On volume integral invariants of non-holonomic dynamical systems, American Journal of Mathematics, 63 (1941), pp. 155-168.

[6] A. Bloch, P. Krishnaprasad, J. Marsden, and R. Murray, Nonholonomic mechanical systems and symmetry, Archive for Rational Mechanics and Analysis, 136 (1996), pp. 21-99.

[7] E. CARTAN, Sur la représentation géométrique des sistème matériels nonholohomes, Proceedings of the International Congress of Mathematicians, 4 (1928), pp. 253-261.

[8] P. Dazord, Mécanique hamiltonienne en présence de constraintes, Illinois Journal of Mathematics, 38 (1994), pp. 148-175.

[9] M. De León, J. C. Marrero, and D. M. De Diego, Vakonomic mechanics versus non-holonomic mechanics: a unified geometrical approach, Journal of Geometry and Physics, 35 (2000), pp. 126-144.

[10] C. Delaunay, Sur la surface de révolution dont la courbure moyenne est constant, Journal de Math'ematiques Pures et Appliquées. Série 1, 6 (1841), pp. $309-320$.

[11] M. Do CARmo, Differential Geometry of Curves and Surfaces, Prentice Hall, 1976.

[12] G. Fusco And W. M. Oliva, Dissipative systems with constraints, Journal of Differential Equations, 63 (1986), pp. 362-388.

[13] G. Gallavotti AND D. Ruelle, SRB states and nonequilibrium statistical mechanics close to equilibrium, Communication in Mathematical Physics, 190 (1997), pp. 279-285.

[14] H. Goldstein, Classical Mechanics, Addison-Wesley Series in Physics, AddisonWesley Publishing Company, Reading, Massachusetts, second ed., 1980.

[15] S. Helgason, Differential Geometry, Lie Groups, and Symmetric Spaces, vol. 80 of Pure and Applied Mathematics, Academics Press, San Diego, 1978.

[16] W. Hoover, Molecular Dynamics, vol. 258 of Lecture Notes in Physics, SpringerVerlag, New York, 1986.

[17] W.-Y. Hsiang AND W.-C. Yu, A generalization of a theorem of Delaunay, Journal of Differential Geometry, 16 (1981), pp. 161-177.

[18] A. Ibort, M. De León, G. Marmo, And D. M. De Diego, Non-holonomic constrained systems as implicit differential equations, preprint, (2001).

[19] K. Kenmotsu, Surface of revolution with prescribed mean curvature, Tôhoku Mathematical Journal, 32 (1980), pp. 147-153.

[20] J. Klein, Espaces variationells et mécanique, Annales de l'Institut Fourier, 12 (1962), pp. 1-124.

[21] M. H. Kobayashi And W. M. Oliva, A note on the conservation of energy and volume in the setting of nonholonomic mechanical systems, Qualitative Theory of Dynamical Systems, 4 (2003), pp. 383-412.

[22] Nonholonomic systems and the geometry of constraints, Qualitative Theory of Dynamical Systems, 5 (2004), pp. 247-259.

[23] J. KOILLER, Reduction of some classical non-holonomic systems with symmetry, Archive for Rational Mechanics and Analysis, 118 (1992), pp. 113-148. 
[24] V. V. Kozlov, Dynamics of systems with non-integrable constraints. I, Moscow University Mechanics Bulletin, 37 (1982), pp. 27-34.

[25] — Dynamics of systems with non-integrable constraints. II, Moscow University Mechanics Bulletin, 37 (1982), pp. 74-80.

[26] Dynamics of systems with non-integrable constraints. III, Moscow University Mechanics Bulletin, 38 (1983), pp. 40-51.

[27] I. Kupka AND W. M. Oliva, The non-holonomic mechanics, Journal of Differential Equations, 169 (2001), pp. 169-189.

[28] C.-M. MARLE, Reduction of constrained mechanical systems and stability of relative equilibria, Communications in Mathematical Physics, 174 (1995), pp. 295318.

[29] - Kinematic and geometric constraints, servomechanisms and control of mechanical systems, Rendiconti del Seminario Matematico dell' Univivesitá e del Politecnico di Torino, 54(4) (1996), pp. 353-364.

[30] - Various approaches to conservative and nonconservative nonholonomic systems, Reports on Mathematical Physics, 42 (1998), pp. 211-229.

[31] E. MASsa AND E. PAGANI, Classical dynamics on non-holonomic systems: a geometric approach, Annales de l'Intitut Henri Poincaré, physique théorique, 55 (1991), pp. 511-544.

[32] K. Nomizu, Ellie Cartan's work on isoparametric families of hypersurfaces, Proceedings of Symposia in Pure Mathematics, 27 (1975), pp. 191-200.

[33] W. M. Oliva, Geometric Mechanics, vol. 1798 of Lecture Notes in Mathematics, Springer-Verlag, 2002.

[34] L. A. PArs, A Treatise on Analytical Dynamics, Heinemann Educational Books, London, 1965.

[35] D. RuelLe, Smooth dynamics and new theoretical ideas in nonequilibrium statistical mechanics, Journal of Statistical Mechanics, 95 (1999), pp. 393-468.

[36] G. Terra And M. H. KobayAshi, On classical mechanical systems with nonlinear constraints, Journal of Geometry and Physics, 49 (2004), pp. 385-417.

[37] A. M. VERSHIK, Classical and nonclassical dynamics with constraints, Lect. Notes Math., 1108 (1984), pp. 278-301.

[38] A. M. VERSHIK AND L. D. FADDEEv, Differential geometry and lagrangian mechanics with constraints, Sov. Phys. Dokl., 17 (1972), pp. 34-36.

[39] _ Lagrangian mechanics in invariant form, Sel. Math. Sov., 1 (1975), pp. 339-350.

[40] R. W. WEBER, Hamiltonian systems with constraints and their meaning in mechanics, Archive for Rational Mechanics and Analysis, 91 (1986), pp. 309-335.

[41] E. T. Whittaker, A Treatise on the Analytical Dynamics of Particles and Rigid Bodies, Cambridge Mathematical Library, Cambridge University Press, Cambridge, UK, fourth ed., 1947.

[42] M. P. WoJTKowski, Magnetic flows and Gaussian thermostats on manifolds of negative curvature, Fundamenta Mathematicae, 163 (2000), pp. 177-191.

[43] D. V. ZENKOV AND A. M. BLOCH, Invariant measures of nonholonomic flows with internal degrees of freedom, Nonlinearity, 16 (2003), pp. 1793-1807. 